\title{
DETERMINAÇÃO DA LACTOSE ANTE ÀS METODOLOGIAS CONTEMPORÂNEAS
}

\section{Lactose determination in relation to contemporary methodologies}

Karina Coelho Moreira da Silva ${ }^{1}$, Gabriel Gama Netto ${ }^{l}$, Louise Bergamin Athayde de Souza ${ }^{l}$, Rodrigo Stephani ${ }^{2}$, Antônio Fernandes de Carvalho ${ }^{1}$, Ítalo Tuler Perrone ${ }^{3 *}$

\section{RESUMO}

A lactose é o açúcar mais abundante em leite e produtos derivados, e com o avanço dos estudos sabe-se que este dissacarídeo não é bem digerido por uma parcela da população, a qual tem como opção consumir produtos reduzidos ou isentos de lactose, sem renunciar aalimentos tão completos. O mercado de produtos isentos de lactose desenvolveu-se muito no Brasil nos últimos anos e a necessidade de métodos confiáveis para determinação do teor final de lactose nos produtos aumentou, por ter se tornado uma determinação obrigatória nas indústrias. Do ponto de vista de segurança ao consumidor e de corresponder ao que a legislação determina, é necessária a adoção de técnicas eficazes de detecção da lactose. Há um universo de possibilidades sendo trabalhadas na pesquisa, ou que já são utilizadas comercialmente. Este trabalho reúne algumas abordagens, como métodos enzimáticos, titulometria, cromatografia, biossensores, glicosímetro e espectroscopia Raman, considerando suas particularidades. Métodos enzimáticos e cromatográficos destacam-se pelos menores limites de detecção e alta repetibilidade.

Palavras-chave: zero lactose; leite; produto lácteo; método analítico.

\begin{abstract}
Lactose is the most abundant sugar of milk and dairy products, and with the progress of studies, it is known that this saccharide is badly digested by a portion

1 Universidade Federal de Viçosa, Departamento de Tecnologia de Alimentos, Viçosa, MG, Brasil.

2 Universidade Federal de Juiz de Fora, Departamento de Química, Juiz de Fora, MG, Brasil.

3 Universidade Federal de Juiz de Fora, Faculdade de Farmácia, Rua José Lourenço Kelmer, s/n, São Pedro, 36036-900, Juiz de Fora, MG, Brasil. E-mail: italotulerperrone@gmail.com

* Autor para correspondência
\end{abstract}


of the population, which has the consumption of reduced or lactose-free products as an option without giving up of such nutritive food. The market for lactose-free products increased during the last years in Brazil and the need for reliable methods for determining the final lactose content in products has increased, as it has become a mandatory determination in the industries. From the point of view of consumer safety and in accordance with the legislation, it is necessary to adopt effective lactose determination techniques. There is a whole world of possibilities being used at research or are already in use commercially. The present study assembles some approaches such as enzymatic methods, titrimetry, chromatography, biosensors, glucometer, and Raman spectroscopy, considering particularities. Enzymatic and chromatographic methods stand out for their lower limits of detection and better repeatability.

Keywords: lactose-free; milk; dairy product; methodology.

\section{INTRODUÇÃO}

Mais que uma tendência, o lançamento de produtos reduzidos ou isentos de lactose tornou-se uma necessidade para marcas que querem se destacar e acompanhar as necessidades de seus consumidores, visto que, atualmente, $68 \%$ da população mundial e $60 \%$ de brasileiros apresenta algum nível de incapacidade de hidrólise deste açúcar (STORHAUG et al., 2017). O mercado no Brasil tem sido promissor, uma vez que 37\% da população aceitaria investir um maior valor nestes produtos em relação aos tradicionais. Esta receptividade foi identificada, e a produção tem se ampliado nos últimos anos, representando $2,5 \%$ em $2016 \mathrm{e} 3,3 \%$ em 2017 , com perspectiva de $4 \%$ até o final de 2018 (EMBRAPA, 2018). A expectativa é de que, até 2020, haja uma ampliação de 15 a $20 \%$ no mercado de produtos zero lactose, segundo levantamento realizado pela Revista Laticínios (2017).

A justificativa para o aquecimento deste mercado de leites especiais é que, mesmo indivíduos cujo organismo apresenta dificuldade em quebrar a lactose, têm a percepção do leite como uma importante fonte de vitaminas e minerais, e os consumidores em geral procuram esses produtos para a adoção de uma rotina mais saudável (NOVOZYMES, 2018a). Outras razões para a ampliação a nível global têm sido estudadas. As correntes de migrações, seja por refúgio ou intercâmbio cultural e acadêmico, mobilizam populações de áreas de alta prevalência de indivíduos intolerantes à lactose para países com apreciável consumo de leite. $\mathrm{O}$ desenvolvimento econômico tem potencial de movimentar esse mercado de leites especiais em países nos quais parte dos habitantes também apresenta esta restrição alimentar. Ademais, é possível destacar a "ocidentalização" na dieta em países asiáticos, como a China, que apresenta uma população altamente intolerante à lactose (HRA, 2017; NOVOZYMES, 2018b, 2018c).

A molécula de lactose é composta pelo resíduo de dois monossacarídeos, glicose e galactose, unidos via ligação glicosídica $\beta-1,4$. É o açúcar mais abundante no leite, e em humanos é clivado pela $\beta$-galactosidase, enzima também chamada delactase. Alguns derivados de leite naturalmente apresentam menor teor de lactose, como queijos maturados e iogurtes, que sofrem fermentação, processo anaeróbio no qual os microrganismos utilizam a lactose como fonte de carbono e geram ácido 
lático. Os consumidores não têm fácil acesso à informação do residual de lactose nestes produtos e,por isso, muitos não se expõem a consumi-los (ACQUARO JÚNIOR et al., 2013; GILLE et al., 2018; MATTAR et al., 2012).

Com o objetivo de instituir um maior controle sobre os alimentos para fins especiais reduzidos ou isentos de lactose, a Agência Nacional de Vigilância Sanitária publicou em fevereiro de 2017 a RDC n ${ }^{\circ} 135$, alterando a já vigente Portaria $n^{\circ} 29$ de janeiro de 1998, que apresentava como objetivo fixar a identidade e características mínimas de qualidade nos alimentos citados. A RDC diminui o limite de $0,5 \mathrm{~g}$ em $100 \mathrm{~g}$ ou $100 \mathrm{~mL}$ do nutriente em referência para $100 \mathrm{mg}$ por $100 \mathrm{~g}$ ou 100 $\mathrm{mL}$, no alimento pronto para consumopara que seja considerado "zero lactose". Para alimentos com reduzido teor, estabeleceu-se a faixa acima de $0,1 \mathrm{~g}$ até $1 \mathrm{~g}$, na mesma porção (BRASIL, 1998, 2017).

A partir de tal mudança na legislação, deve-se aumentar o rigor no controle de processos por meio da garantia de qualidade, sendo necessário buscar métodos analíticos cada vez mais precisos, exatos e acessíveis. Os produtos oriundos diretamente da hidrólise da lactose, bem como as moléculas geradas a partir da glicose e galactose (oligossacarídeos) atuam como interferentes nos métodos analíticos para quantificação da lactose (FAI; PASTORE, 2015; GILLE et al., 2018; ERICH et al., 2012).

O presente trabalho apresentou o método enzimático, a titulometria, a cromatografia, o biossensor e o glicosímetro para determinação de lactose.A titulometria, o método enzimático, o glicosímetro e o biossensor foram escolhidos por se tratarem de metodologias simples e de baixo custo podendo ser facilmente implementadas nas indústrias de laticínios, desta forma representa a realidade de muitas fábricas no tocante a dosagem de açúcares. A cromatografia foi escolhida por ser atualmente o método de referência empregado pelo Ministério da Agricultura Pecuária e Abastecimento para quantificação de lactose em produtos hidrolisados, bem como pelo grande número de trabalhos descritos na literatura sobre a aplicação desta técnica para quantificação de açúcares. O objetivo deste artigo foi levantar as principais informações publicadas na literatura sobre estes métodos analíticos empregados para determinação da lactose, apontando suas potencialidades e pontos fracos, principalmente quando aplicados na determinação em lácteos e lácteos lactose hidrolisados.

\section{REFERENCIAL TEÓRICO}

\section{Métodos enzimáticos}

São métodos em que o substrato de interesse sofre ação de uma ou mais enzimas e o produto é quantificado através de equipamentos como o espectrofotômetro. $\mathrm{O}$ princípio deste método em relação à lactose é a hidrólise em seus açúcares mais simples pela $\beta$-galactosidase (Figura 1). Em sequência, o analista promove uma reação de oxirredução, na qual o agente oxidante nicotinamida-adenina $\left(\mathrm{NAD}^{+}\right)$é reduzido a NADH e a D-galactose é oxidada a ácido D-galacturônico. É possível, então, determinar a concentração de lactose pela relação estequiométrica com o NADH formado, que é o produto de interesse na leitura por espectrofotometria a $340 \mathrm{~nm}$. Os reagentes e tampões necessários para esse processo são em geral vendidos em kits, que podem ou não conter outros reagentes, a fim de otimizara análise. É o método oficial da AOAC (CUNNIF, 1998) para determinação de lactose em leite, o que passa confiabilidade nos resultados a nível internacional.

É uma técnica complexa, dispendiosa e dependendo do fabricante, demorada. Os 
kits são importados, o que pede um excelente planejamento para a compra do material e, se houver perda de reagentes na execução, é necessário comprar todo o kit novo. Não há compatibilidade com outros fabricantes para que seja feita a substituição de um insumo (ACQUARO JÚNIOR et al., 2013). A Tabela 1 mostra uma comparação das especificações de dois fabricantes de kits de teste enzimático para análise de lactose.

Blais (1993) e Blais; Vailhen (1995) trabalharam com uma modificação do método a fim de contornar as desvantagens do mesmo. $\mathrm{Na}$ etapa inicial permanece a hidrólise da lactose em seus dois dissacarídeos pela lactase e, em seguida, conversão da glicose em D-glucono-1,4-lactona e peróxido de hidrogênio, através de glicose oxidase e na presença de oxigênio. O peróxido de hidrogênio é então mensurado por reação com iodeto gerando iodo, que forma um complexo colorido com álcool polivinílico. $\mathrm{O}$ ensaio foi realizado em placa de microtítulo e a leitura realizada em equipamento específico para este tipo de placa. Os resultados com a nova metodologia foram positivamente comparáveis com os do kit tradicional e o novo sistema de detecção apresentou uma relação linear de resposta à concentração de lactose presente em amostras de queijo parmesão. Porém, mais ensaios seriam necessários para melhorar a precisão e avaliar a replicabilidade em outras matrizes alimentícias.

Autores anteriores a seu tempo também buscavam métodos enzimáticos que fossem eficazes para a quantificação de lactose em

\section{Lactose $+\mathrm{H}_{2} \mathrm{O} \stackrel{\text { p-galactosidase }}{\longrightarrow}$ D-galactose + D-glicose (1)}

$\alpha$-D-Galactose $\underset{\text { mutarotase }}{\longrightarrow} \boldsymbol{\beta}$-D-Galactose (2)

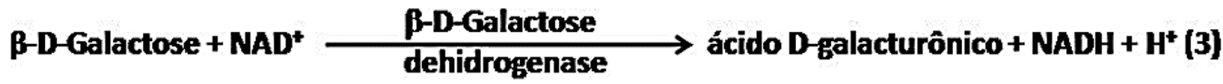

Figura 1 - Reações químicas do método enzimático de determinação de lactose (MEGAZYME, 2011)

Tabela 1-Critérios de análise em testes enzimáticos

\begin{tabular}{ccc}
\hline Características & Megazyme & R-Biopharm \\
\hline $\begin{array}{c}\text { Menor unidade de } \\
\text { absorvância diferenciável } \\
\text { Menor concentração } \\
\text { de lactose }\end{array}$ & 0,010 & 0,005 \\
$\begin{array}{c}\text { Menor concentração } \\
\text { de D-galactose }\end{array}$ & $\begin{array}{c}1,48 \mathrm{mg} / \mathrm{L} \mathrm{em} 1 \mathrm{~mL} \mathrm{de} \\
\text { amostra }\end{array}$ & $\begin{array}{c}2 \mathrm{mg} / \mathrm{L} \mathrm{em} 0,5 \mathrm{mLde} \\
\text { amostra }\end{array}$ \\
$\begin{array}{c}\text { Limite de detecção } \\
\text { Faixa de linearidade }\end{array}$ & $\begin{array}{c}\text { amostra } \\
\text { amg } / \mathrm{L} \mathrm{em} 1 \mathrm{~mL} \mathrm{de}\end{array}$ & $\begin{array}{c}1 \mathrm{mg} / \mathrm{Lm} 0,5 \mathrm{~mL} \mathrm{de} \\
\text { amostra }\end{array}$ \\
\hline
\end{tabular}

Fonte: R-Biopharm (2015); Megazyme(2011). 
amostras. Kleyn e Trout (1984) realizaram um trabalho em colaboração com 8 indústrias de alimentos para determinação de lactose em oito amostras desconhecidas de leite integral, desnatado e semidesnatado, mas obtiveram resultados baixos em relação à técnica oficial da época.

\section{Métodos titulométricos}

São métodos para determinação de açúcares totais redutores e não redutores não específicos para nenhum tipo de açúcar. Desta forma, não são recomendados para produtos hidrolisados, uma vez que são inespecíficos. Utilizam reagentes e vidrarias comuns a diversos laboratórios, porém mais suscetíveis ao acúmulo de erros, pois apresentam muitas etapas na metodologia.

\section{Método de Lane-Eynon}

O método de Lane-Eynon (LE) baseia-se na determinação do volume de amostra necessário para reduzir uma medida da solução de Fehling, de concentração conhecida. É possível identificar o ponto final da titulação através do uso de indicador, como azul de metileno. A solução de Fehling é formada por íons bivalentes de cobre $\left(\mathrm{Cu}^{+2}\right)$ complexados com tartarato em meio alcalino. Neste meio, os carboidratos são oxidados a ácidos aldônicos, formando óxido de cobre I (precipitado vermelho-tijolo) (RAJAKYLÄ; PALOPOSKI, 1983).

Tempo de aquecimento, temperatura e concentração dos reagentes devem ser bem controlados para a execução do método de LE. A amostra necessariamente deve ser neutra e nenhum agente redutor deve estar presente na matriz a fim de não haver alteração nos resultados (BIRCH, 1985). A ebulição deve ser mantida na análise para evitar que o óxido cuproso entre em contato com o oxigênio do ar e seja oxidado, retornando à forma de óxido cúprico. O aquecimento prolongado pode decompor os açúcares da amostra, portanto a titulação deve ser realizada em, no máximo, três minutos (TAVARES et al., 2010).

Esta técnica foi empregada em trabalhos que têm como objetivo verificar adequação à legislação, no estudo de leites fluidos comercializados em São Luiz do Maranhão, por Silva e sua equipe (2014); e na investigação de fraude por sacarose em leite in natura (necessitou inversão da sacarose na metodologia), proveniente de produtores de Passo Fundo, RS, realizada por Tonial e Gobbi em 2015. Não foi encontrado na literatura o limite de detecção da técnica.

\section{Método da Cloramina-T}

$\mathrm{O}$ método da Cloramina-T foi desenvolvido como alternativa ao método de Lane-Eynon, dispensando o trabalho a quente e facilitando a percepção do ponto de viragem. A amostra é desproteinizada com ácido wolfrâmico e, posteriormente, a reação entre a lactose e a Cloramina-T adicionada de iodeto de potássio libera iodo reduzido, que é quantificado por iodometria (WOLFSCHOON-POMBO; CASAGRANDE, 1982).

Muitos autores a utilizaram em seus experimentos, como Pereira et al. em 2010 ao descrever a taxa de cristalização da lactose em soro concentrado por meio de cristalizador de bancada; e a equipe de Renhe (2018), na caracterização físico-química de leite condensado.

\section{Métodos Cromatográficos}

Cromatografia Líquida de Alta Performance

A Cromatografia Líquida de Alta Performance, ou ainda, HPLC (do inglês, High Performance Liquid Chromatography), tem sido explorada em trabalhos por apresentar, de forma geral, sensibilidade, riqueza de resultados, utilizar pouca amostra, detectar 
e quantificar não apenas lactose, mas outros sacarídeos simultaneamente, e ser uma técnica automatizada (GALANT et al., 2015; RESENDE; MATOS, 2009; SEXTON, 2004; TROIANO et al., 2016). Através da migração de uma amostra dispersa em uma fase móvel líquida, ocorre a separação de seus componentes por interação com a fase estacionária e ao passar pelo detector, o sinal gerado é proporcional à concentração dos analitos, permitindoa separação e a quantificação através desta técnica.

Alguns autores utilizam acetonitrila ou metanol (FREITAS et al., 2017; PERATI et al., 2003; RESENDE; MATOS, 2009; SEXTON, 2004; TROIANO et al., 2016)como fase móvel na HPLC, o que traz riscos para o analista (PUBCHEM, 2018a, 2018b). Outros trabalhos, como o de Acquaro Júnior. et al. em 2013 e o de Naegele em 2016, utilizam água ultrapura como fase móvel, o que também traz praticidade para a utilização em grandes quantidades. Os outros solventes já mencionados precisam ser constantemente adquiridos e necessitam de permissão da Polícia Federal, no caso da acetonitrila (BRASIL, 2018).

Acquaro Júnior e seus colaboradores em 2013trabalharam no desenvolvimento e validação de uma metodologia que permitisse a extração e determinação de lactose baseada em princípios da Química Verde (reagentes de fácil obtenção, custo relativamente baixo e baixa toxicidade). A extração em amostras de leite pasteurizado foi realizada com etanol, o conteúdo foi ressuspenso com água ultrapura e levado para análise por HPLC com detector de índice de refração. O limite de detecção para a lactose estabelecido foi de $0,05 \mathrm{~g} / \mathrm{L} \mathrm{e}$ o limite de quantificação de $0,15 \mathrm{~g} / \mathrm{L}$.

\section{Cromatografia de Íons}

O Manual de Métodos Oficiais para Análise de Alimentos de Origem Animal estabelece o método por cromatografia de troca iônica para detecção de lactose em uma ampla gama de produtos lácteos, exceto caseinatos e queijo em pó. É utilizada uma coluna de estireno/divinilbenzeno em um cromatógrafo de íons, detector amperométrico de célula de ourono modo PAD (detecção amperométrica pulsada, do inglês, Pulsed Amperometric Detection) e como fase móvel é utilizada uma solução de hidróxido de sódio a $5 \mathrm{mmol} / \mathrm{L}$ (BRASIL, 2018).

O trabalho de Barragan (2016) foi voltado para a melhoria na detecção de carboidratos em cromatógrafo de íons, modificando a detecção. Na comparação entre pulsos de detecção, os limites encontrados foram equivalentes, sendo 13,4 $\mu \mathrm{g} / \mathrm{Lpara}$ a atual tecnologia utilizada e $14,7 \mu \mathrm{g} / \mathrm{L}$ para a nova possibilidade (SAD, detecção amperométrica por varredura, do inglês, Scanning Amperometric Detection).

\section{Biossensores}

Biossensores utilizam como princípio reações que ocorreriam na natureza, aplicadas a um equipamento que detecta e traduz o sinal produzido pelo composto de interesse em contato com a biocamada. Para isto, um componente biológico, como uma enzima, anticorpo ou ácido nucléico, é empregado. A estrutura de um biossensor conta com o compartimento de amostra, o sistema de reconhecimento (que tem contato direto com a amostra), um transdutor (traduz a reação em um sinal mensurável) e processador de sinal, que filtra, amplifica, analisa e transfere o sinal para um monitor (CALIL; SILVA, 2011). Há grande cenário para emprego de biossensores na detecção de açúcares, e o principal é a glicose, mas há também aplicações na análise de contaminantes, no acompanhamento de fermentações e na garantia da qualidade de alimentos (DORNEMANN, 2016).

Como mencionado, biossensores podem apresentar diversos tipos de materiais em sua composição, e este universo de 
possibilidades aplica-se também à detecção de lactose. Salvo-Comino e seus colaboradores (2018) trabalharam em um multissensor de ftalocianina de cobre sulfonado (camada aniônica), tetrafluoroborato de 1-butil-3metilimidazólio com quitosana (camada catiônica) e galactose oxidase imobilizada, e realizaram testes em amostras de leite com diferentes concentrações de lactose e gordura. Também obtiveram êxito na determinação da acidez, proteínas e gorduras, de maneira simples e rápida e sem necessidade de prétratamento da amostra.

Trabalhando com outros materiais de base (polímeros e eletrodo de platina), a enzima galactose oxidase e a $\beta$-D-galactosidase foram exploradas por Gursoy e colaboradores, em 2018, obtendo um limite de detecção de $14 \mu \mathrm{M}$. Focados no desafio por produtos reduzidos em lactose (que apresentam níveis aumentados de glicose e galactose), Caron e Trowell, em 2018, trabalharam em um biossensor que superasse $o$ método de cromatografia líquida, que precisa ser muito bem estruturado para separar os componentes em uma matriz tão complexa quanto à do leite, e também se destacar em relação abiossensores enzimáticos, que podem ser mais demorados e apresentar baixa sensibilidade. A abordagem utilizada baseouse em um regulador de transcrição encontrado em Clostridium perfringens que é altamente seletivo à lactose. $\mathrm{O}$ sensor apresentou-se duzentas vezes mais sensível ao dissacarídeo que aos resíduos de sua hidrólise, com limite de detecção de $0,2 \mu \mathrm{M}$, sem a necessidade de remover previamente gordura e proteínas.

Biossensores ainda mais complexos apresentam soluções para a detecção de lactose em matrizes mais diversas, como iogurte, manteiga, queijo e maionese, sem a necessidade de prévia retirada de interferentes. Conzuelo e sua equipe, em 2010, trabalharam em um eletrodo baseado em uma monocamada de ácido 3-mercaptopropiônico, enzimas $\beta$-galactosidase, glicose oxidase, peroxidase em ouro modificado e um mediador, coimobilizados em uma membrana de diálise. O princípio da técnica é a hidrólise da lactose e a rápida oxidação da glicose a ácido glucônico e água oxigenada, que é reduzida pela peroxidase.

Todos estes trabalhos encontrados na literatura voltados para produtos com redução de lactose são bastante recentes, mas comercialmente existem biossensores já disponíveis, como o Biomilk 300 (BIOLAN; DSM, 2015), que utiliza enzimas e componentes eletroativos, que geram um sinal percebido pelo transdutor amperométrico, e pode ser utilizado para diversos produtos lácteos, com exceção de manteiga, por não ser miscível em solução aquosa. Dependendo da calibração realizada, o equipamento alcança $50 \mathrm{mg} / \mathrm{L}$ como limite de detecção.

Apesar de parecer complexo o sistema de reconhecimento e as reações envolvidas entre eletrodo e a amostra, os biossensores trazem facilidade para o operador e rapidez de resposta, alinhando a sensibilidade e baixo limite de detecção.

\section{Glicosímetro}

O glicosímetro é o equipamento comumente utilizado por indivíduos diabéticos para checagem do nível da glicemia sanguínea. Os medidores disponíveis no mercado utilizam fitas que promovem reações bioquímicas com a amostra de sangue. Podem diferir-se no tipo de detector usado; aqueles que seguem o princípio da fotometria avaliam a amostra por mudança de intensidade da sua cor, enquanto os amperométricos detectam a intensidade da corrente elétrica que passa pela amostra. A sua adaptação ao uso em produtos lácteos é justificada por sua praticidade, rapidez e eficiência na quantificação de lactose (MACIEL, 2014). A faixa de medição do método é de 10 a $600 \mathrm{mg} / \mathrm{dL}$ (ACCU-CHECK, 2018). 
Campos et al. em 2014 realizaram ensaios para comparação com método tradicional de determinação de lactose e obtiveram êxito em seu experimento. Nos ensaios de acompanhamento de hidrólise da lactose, o equipamento respondeu conforme esperado, ao aumento da concentração de glicose e galactose no meio, mas isso também torna o resultado difícil de ser interpretado, visto que o método não é diferencial entre a lactose e os açúcares formados após a hidrólise. Em matrizes mais complexas, com adição de glicose, por exemplo, torna-se impossível a verificação da concentração da lactose sem algum tratamento complementar da amostra. Apesar de se observar que a gordura subestima os valores mensurados, a diferença não é suficiente para que não sejam admitidos os mesmos.

No trabalho de Metzger, do centro de pesquisa Midwest Dairy Foods, em 2013, foi estudado o uso de glicosímetro como um método de baixo custo e que exige mínimo treinamento para que operadores possam utilizar em linha. Diferentemente do trabalho de Campos, alguns cuidados foram tomados, como a prévia hidrólise enzimática de lactose da amostra de soro e queijos processados. Demonstrou-se que o método foi eficaz do ponto de vista abordado, e retornava com acurácia a concentração de lactose.A faixa de medição do método é de 20 a 600 mg/dL, conforme descrito no manual do equipamento (RELION, 2009).

\section{Espectroscopia RAMAM}

A espectroscopia RAMAN baseia-se nas propriedades de espalhamento de radiação eletromagnética monocromática da matéria, de forma que a frequência propagada é diferente da que foi incidida. Para isso, a instrumentação desta técnica conta com laser como radiação incidente e confiável para o método, por ser direcional e monocromático. Também conta com detectores altamente capazes, como os multicanal, microscópio óptico acoplado e fibra ótica (ALMEIDA, 2011). É uma técnica que tem tido notoriedade na área de alimentos por apresentar clareza nos resultados (bandas estreitas e bem resolvidas); pela possibilidade de realizar análises não destrutivas, sem complexo preparo das amostras, uso de reagentes e geração de subprodutos tóxicos;por permitir a determinação de mais de um componente simultaneamente e pelo fato da água não interferir nos espectros (ALMEIDA, 2011; YANG; YING, 2011).

Têm-se dado atenção a esta técnica na obtenção do espectro de carboidratos. No trabalho de Arboleda e Loppnow (2000) foi possível a obtenção do espectro de diversos monossacarídeos, em concentrações abaixo de 10 mM. No trabalho de Almeida, em 2011, a espectroscopia RAMAN foi uma ferramenta essencial para avaliar qualidade de leite em pó e leite condensado rapidamente. Foi possível identificar adulterações com soro ou amido acima de 5\% e a modificação do leite em pó no armazenamento devido à cristalização da lactose. Para soro de leite concentrado também é possível este monitoramento da lactose, que modifica-se do estado amorfo ao cristalino durante processamento e sheflife (STEPHANI, 2015; STEPHANI et al., 2017). RAMAN pode ser empregada para acompanhar produção e vida de prateleirade produtos com redução da lactose (STEPHANI et al., 2016; TORRES et al., 2017), e também é possível verificar fraudes com maltodextrina em leite em pó tradicional ou com redução de lactose (RODRIGUES JÚNIOR et al., 2016).

\section{Breve consideração sobre as metodologias}

Após o levantamento de algumas técnicas que estão sendo trabalhadas na literatura, é possível sumariar na Tabela 2 o que foi observado, destacando em breves Vantagens x Desvantagens. 


\section{CONSIDERAÇÕES FINAIS}

Técnicas titulométricas demonstram-se complexas e pouco eficientes para o baixo limite de detecção necessário à análise em produtos isentos ou com baixa concentração de lactose. Metodologias atualizadas têm grande aplicabilidade neste cenário, além de serem menos complexas e exigirem menor tempo de preparação para as amostras. A fim de adequar-se ao limite adotado pela legislação brasileira, as indústrias devem recorrer a estes métodos que trazem segurança nos resultados, devido à sensibilidade, repetitividade e seletividade, dentre os atributos específicos de cada técnica e que atendam a cada demanda.

\section{REFERÊNCIAS}

ACCU-CHECK. Monitor de Glicemia. Manual de Instruções. Disponível em: https:// debemcomavida.accuchek.com.br/media/6114/ manual_active.pdf Acesso em: 16 set 2018.
ACQUARO JÚNIOR, V. R. et al. Desenvolvimento e validação de método para extração e quantificação através de HPLC com índice de refração para lactose em leite pasteurizado. Scientia Chromatographica, v. 5 , n. 2 , p. 137-145, 2013.

ALMEIDA, M. R. Avaliação da qualidade e variedade de leite em pó e leite condensado por espectroscopia Raman e análise multivariada. 2011. Dissertação (Mestrado em Química) - Departamento de Química, Universidade Federal de Juiz de Fora, Juiz de Fora, 2011.

ARBOLEDA, P. H.; LOPPNOW, G. R. Raman spectroscopy as a discovery tool in carbohydrate chemistry. Analytical Chemistry, v. 72, n. 9, p. 2093-2098, 2000.

CUNNIFF, P. A. (ed.). Official methods of analysis of AOAC International. 16th ed. 4th rev. Gaithersburg: AOAC International, 1998.

Tabela 2 - Vantagens e desvantagens em técnicas de detecção de lactose

\begin{tabular}{|c|c|c|}
\hline Técnica & Vantagens & Desvantagens \\
\hline Enzimática & Baixo limite de detecção. & Dispendiosa e demorada. \\
\hline Titulométrica & $\begin{array}{l}\text { Reagentes e vidrarias disponíveis } \\
\text { na maioria dos laboratórios. }\end{array}$ & $\begin{array}{l}\text { Complexa, não seletiva, } \\
\text { passível de erros grosseiros. }\end{array}$ \\
\hline Cromatográfica & $\begin{array}{l}\text { Automatizado, alta sensibilidade, } \\
\text { repetitividade, baixo limite de } \\
\text { detecção, pureza na separação e ampla } \\
\text { faixa de utilização. }\end{array}$ & $\begin{array}{l}\text { Exige preparação da amostra, } \\
\text { grande tempo de estabilização } \\
\text { do equipamento e dispendioso. }\end{array}$ \\
\hline Biossensor & $\begin{array}{l}\text { Alta seletividade, compacto, baixo } \\
\text { limite de detecção, não necessita } \\
\text { preparo da amostra. }\end{array}$ & $\begin{array}{l}\text { Não é automático, tempo de } \\
\text { vida útil limitado em alguns } \\
\text { casos, caro. }\end{array}$ \\
\hline Glicosímetro & Praticidade, acessibilidade financeira. & $\begin{array}{l}\text { Não é seletivo (excesso de } \\
\text { glicose interfere), alto limite } \\
\text { de detecção. }\end{array}$ \\
\hline $\begin{array}{l}\text { Espectroscopia } \\
\text { RAMAN }\end{array}$ & $\begin{array}{l}\text { Não destrutivo, sem pré-tratamento } \\
\text { de amostra, ampla faixa de utilização. }\end{array}$ & Resultado difícil de interpretar \\
\hline
\end{tabular}


R-BIOPHARM. Lactose/D-Galactose. UVmethod for the determination of lactose and D-galactose in foodstuffs and other materials. Cat. No. 10176303035. Disponível em: https://www.azmax.co.jp/wp-content/ uploads/2015/11/Lactose-Galactose EN_10176303035_2014-02.pdf Acesso em: 29 set 2018 .

BARRAGAN, J. T. C. Melhorias nos sistemas de detecção de carboidratos para cromatografia de íons. 2016. $154 \mathrm{f}$. Tese (Doutorado em Ciências) - Instituto de Química, Universidade Estadual de Campinas, Campinas. 2016.

BIOLAN; DSM. Data Sheet Biomilk 300 Biosensor for analysing lactose in lactose free dairy products. 2015. Disponível em: http:// www.biolanmb.com/sectors/biomilk-dairyproduct-sensorsAcesso em: 09 out 2018.

BIRCH, G. G. (ed.). Analysis of Food Carbohydrates. New York: Elsevier Applied Science Publishers, 1985.

BLAIS, B. W. An automated microassay for lactose in foods employing a simple and inexpensive enzymatic detection system. MAN Microbiologie-Aliments-Nutrition, v. 11, p. 43-49, 1993.

BLAIS, B. W.; VAILHEN, C. A novel enzymatic microassay for the determination of lactose in grated parmesan cheese. Food Control, v. 6, n. 4, p. 215-217, 1995.

BRASIL. Ministério da Agricultura, Pecuária e Abastecimento. Secretaria de Defesa Agropecuária. Manual de Métodos Oficiais para Análise de Alimentos de Origem Animal. 1. ed. Brasília: MAPA, 2018. 140 p. ISBN 978-85-7991-111-8.

BRASIL. Ministério da Justiça e Segurança Pública. Polícia Federal. Anexo 1. Lista dos produtos químicos controlados. Disponível em: http://www.pf.gov.br/servicos-pf/ produtos-quimicos/legislacao/anexos-daportaria-1274-03/01-Anexo I - Listas.pdf/view Acesso em: 30 set. 2018.

BRASIL. Ministério da Saúde. Agência Nacional de Vigilância Sanitária. Portaria $\mathrm{n}^{\mathrm{o}} 29$, de 13 de janeiro de 1998. Aprova o Regulamento Técnico referente a Alimentos para Fins Especiais. Diário Oficial da União: seção 1, Brasília, DF, n. 60, p. 2, 30 mar. 1998.

BRASIL. Ministério da Saúde. Agência Nacional de Vigilância Sanitária. Resolução $\mathrm{RDC} \mathrm{n}^{\circ} 135$, de 08 de fevereiro de 2017. Altera a Portaria SVS/MS n ${ }^{\circ} 29$, de 13 de janeiro de 1998, que aprova o regulamento técnico referente a alimentos para fins especiais, para dispor sobre os alimentos para dietas com restrição de lactose. Diário Oficial da União: seção 1, Brasília, DF, n. 29, p. 44, 09 fev. 2017.

CALIL, S. S.; SILVA, P. R. Q. Biossensores: estrutura, funcionamento e aplicabilidade. In: MOSTRA DE PRODUÇÃO CIENTÍFICA DA PÓS-GRADUAÇÃO LATO SENSU DA PUC GOIÁS, 6., 2011, Goiânia. Artigos [...]. Goiânia: PUC Goiás, 2011. p. 1-20.

CAMPOS, N. S. et al. Lactose quantification in dairy products by Accu-Chek ${ }^{\circledR}$ glucometer. Revista Virtual de Química, v. 6, n. 6, p. 1677-1686, 2014.

CARON, K.; TROWELL, S. C. Highly sensitive and selective biosensor for a disaccharide based on an AraC-like transcriptional regulator transduced with bioluminescence resonance energy transfer. Analytical Chemistry, v. 90, n. 21, p. 12986-12993, 2018.

CONZUELO, F. et al. An integrated amperometric biosensor for the determination of lactose in milk and dairy products. Journal of Agricultural and Food Chemistry, v. 58, 
n. 12, p. $7141-7148,2010$.

DORNEMANN, G. M. Comparação de métodos para determinação de açúcares redutores e não-redutores. 2016. Trabalho de Diplomação (Graduação em Engenharia Química) - Departamento de Engenharia Química, Universidade Federal do Rio Grande do Sul, Porto Alegre, 2016.

EMBRAPA GADO DE LEITE. Intolerância à lactose:bom para os negócios. In: MARTINS, P. C. et al. (coord.). Anuário Leite 2018: Indicadores, tendências eoportunidadespara quem vive no setor leiteiro. São Paulo: Texto Comunicação Corporativa, 2018.p. 62.

ERICH, S.; ANZMANN, T.; FISCHER, L. Quantification of lactose using ion-pair RPHPLC during enzymatic lactose hydrolysis of skim milk. Food chemistry, v. 135, n. 4, p. 2393-2396, 2012.

FAI, A. E. C.; PASTORE, G. M. Galactooligossacarídeos: Produção, Benefícios à saúde, aplicação em alimentos e perspectivas. Scientia Agropecuaria, v. 6, n.1, p. 69-81, 2015.

FREITAS, M. C. et al. Desenvolvimento de metodologia para análise de amostras lácteas com teor reduzido de lactose utilizando HPLC-ELSD. In: CONGRESSO INTERINSTITUCIONAL DE INICIAÇÃO CIENTÍFICA, 11., 2017, Campinas. Anais [...]. Campinas: Apta Regional, 2017.

GALANT, A. L.; KAUFMAN, R. C.; WILSON, J. D. Glucose: Detection and analysis. Food Chemistry, v. 188, p. 149-160, 2015.

GILLE, D. et al. Detection of lactose in products with low lactose content. International Dairy Journal, v. 83, p. 17-19, 2018.

GURSOY, O. et al Development of a new two-enzyme biosensor based on poly(pyrrole- co-3,4-ethylenedioxythiophene) for lactose determination in milk. Polymer Engineering \& Science, v. 58, n. 6, p. 839-848, 2018.

HRA FOOD \& DRINK CONSULTANTS. Lactose-free: Developments and trends in the EU market. [s. 1.]: HRA Food \& Drink, 2017. Disponível em: https:/hra-global.com/ insights-and-reports/european-lactose-freemarket-report-2017-2018/Acessoem: 24 jan. 2018.

KLEYN, D. H.; TROUT, J. R. Enzymaticultraviolet method for measuring lactose in milk: collaborative study. Journal Association of Official Analytical Chemists, v. 67, n. 3, p. 637-40, 1984.

MACIEL, T. E. S. Correlação de medidas de glicosímetros e dosagem laboratorial. 2014. 67 f. Dissertação (Mestrado em Engenharia Biomédica) - Universidade Tecnológica Federal do Paraná, Curitiba, 2014.

MATTAR, R.; MAZO, D. F. C.; CARRILHO, F. J. Lactose intolerance: Diagnosis, genetic, and clinical factors. Clinical and Experimental Gastroenterology, v. 5, p. 113, 2012.

MEGAZYME. Microplate Format Assay Procedure for Lactose and D-Galactose (Rapid). 2011. Disponível em: https://secure. megazyme.com/files/Other_automated_assay_ procedures/K-LACGAR_ALT.pdf Acesso em: 10 out 2018 .

METZGER, L. Rapid measurement of the lactose content of cheese whey and process cheese using a commercially available blood glucose meter. Agricultural Utilization Research Institute, p. 0-10, 2013.

NAEGELE, E. Analysis of lactose free dairy products using the Agilent 1220 Infinity LC with Evaporative Light Scattering Detection. 2016. Disponível em: https://www. 
agilent.com/cs/library/applications/59912252EN.pdf Acesso em: 27 set. 2018.

\section{NOVOZYMES. Regional opportunities in} low lactose. Disponível em: https://dairylab. novozymes.com/regional-opportunities-inlow-lactose Acesso em: 14 jan. 2018a.

NOVOZYMES. Report: The market for zero-lactose in Brazil. Disponível em: https:// dairylab.novozymes.com/report-zero-lactoseopportunities-in-brazil Acesso em: 14 jan. 2018b.

NOVOZYMES. Top trends driving lactosefree opportunities. Disponível em: https:// dairylab.novozymes.com/trends-drivinglactosefree-opportunities Acesso em: 14 jan. $2018 \mathrm{c}$.

PERATI, P.; BORBA, B.; ROHRER, J. Determination of lactose in lactose-free milk products by high-performance anionexchange chromatography with pulsed amperometric detection. 2003. Disponível em: https://assets thermofisher.com/TFSAssets/CMD/Application-Notes/AN-248-ICLactose-Milk-AN70236-EN.pdf. Acesso em: 28 set. 2018.

PEREIRA, J. P. F. et al. Percentual de cristalização da lactose em cristalizador de bancada de soro de leite concentrado. Revista de Instituto de Laticínios Cândido Tostes, v. 65 , p. 26-32, 2010.

PUBCHEM OPEN CHEMISTRY DATABASE. Compound Summary -Acetonitrile. Disponível em: https://pubchem.ncbi.nlm. nih.gov/compound/acetonitrile\#section=Top Acesso em: 30 set. $2018 \mathrm{a}$.

PUBCHEM OPEN CHEMISTRY DATABASE. Compound Summary - Methanol. Disponível em:https://pubchem.ncbi.nlm.nih.gov/ compound/887 Acesso em: 30 set. 2018 b.
RAJAKYLÄ, E.; PALOPOSKI, M. Determination of sugars (and betaine) in molasses by high-performance liquid chromatography: Comparison of the results with those obtained by the classical lane-eynon method. Journal of chromatography $\mathbf{A}$, v. 282, p. 595-602, 1983.

RELION. ReliOn Confirm Owner's Manual. 2009. Disponível em: https://www. manualagent.com/relion/relion-confirm/ owners-manual Acesso em: 10 set. 2018.

RENHE, Í. R. T. et al. Characterization of physicochemical composition, microbiology, sensory evaluation and microscopical attributes of sweetened condensed milk. Food Science and Technology, v. 38, n. 2, p. 293-298, 2018.

RESENDE, M. F.; MATOS, M. A. C. Determinação de galactose, glicose e lactose em leite por HPLC. In: REUNIÃO ANUAL DA SOCIEDADE BRASILEIRA DE QUÍMICA, 33.,2009, Juiz de Fora. Anais [...]. Juiz de Fora: UFJF, 2009.

REVISTA LATICÍNIOS. Nova lactase traz inovação para produção de produtos zero lactose. Revista Indústria de Laticínios. 2017. Disponível em: http://revistalaticinios.com.br/ noticias/empresas-e-negocios/nova-lactasetraz-inovacao-para-producao-de-produtos-zerolactose Acesso em: 12 dez. 2017.

RODRIGUES JÚNIOR, P. H. et al. FTRaman and chemometric tools for rapid determination of quality parameters in milk powder: Classification of samples for the presence of lactose and fraud detection by addition of maltodextrin. Food Chemistry, v. 196, p. 584-588, 2016.

SALVO-COMINO, C. et al. Discrimination of milks with a multisensor system based on layer-by-layer films. Sensors, v. 18, n. 8, p. 2716, 2018. 
SEXTON, D. L. Determination of lactose by Reversed-Phase High Performance Liquid Chromatography. 2004. Tese (Master of Science in Chemistry) - Department of Chemistry, East Tennessee State University, Johnson City, 2004.

SILVA, M.V.V. et al. Determinação do teor de lactose em leite flúido pelo método LaneEynon. In: CONGRESSO BRASILEIRO DE QUÍMICA, 54., 2014, Natal. Anais [...]. Natal: ABQ, 2014. Disponível em: http://www.abq. org.br/cbq/2014/trabalhos/3/5829-19482.html Acesso em: 20 set.2018.

STEPHANI, R. Uma contribuição à pesquisa das propriedades químicas dos concentrados proteicos de soro do leite. 2015. Tese (Doutorado em Química) - Instituto de Ciências Exatas, Universidade Federal de Juiz de Fora, Juiz de Fora, 2015.

STEPHANI, R. et al. Use of Raman spectroscopy to identify modification of reduced and low lactose milk powder during shelf life. In: IDF CONCENTRATION AND DRIED MILK PRODUCTS SYMPOSIUM. Dublin: IDF, 2016.

STEPHANI, R. et al. Raman spectroscopy as a tool to identify modification of whey protein concentrate (WPC) during shelf life. Food Packaging and Shelf Life, v. 11, p. 1-9, 2017.

STORHAUG, C. L.; FOSSE, S. K.; FADNES, L. T. Country, regional, and global estimates for lactose malabsorption in adults: A systematic review and meta-analysis. The Lancet Gastroenterology and Hepatology, v. 2, n. 10, p. 738-746, 2017.
TAVARES, J. T. Q. et al. Interferência do acido ascórbico na determinação de açúcares redutores pelo método de Lane e Eynon. Química Nova, v. 33, n. 4, p. 805-809, 2010.

TONIAL, T.; GOBBI, D. L. Investigação de fraudes em leite in natura por sacarose e determinação da lactose pelo método de LaneEynon. In: SEMANA DO CONHECIMENTO UPF, 2015, Passo Fundo. Anais [...]. Passo Fundo: UPF, 2015.

TORRES, J. K. F. et al. Technological aspects of lactose-hydrolyzed milk powder. Food Research International, v. 101, p. 45-53, 2017.

TROIANO, R.; DENARO, F.; REUTER, W. M. The analysis of lactose in milk and cheese products by HPLC with RI detection. Walthan: Perkin Elmer, 2016. Disponível em: https://www.perkinelmer.com/lab-solutions/ resources/docs/APP_The_Analysis_of Lactose_in_Milk_and_Cheese_Products_by_ HPLC_Note_012755_01 (1).pdf. Acesso em: 18 set. 2018 .

W O L F S C H O O N - P O M B O, A . ; CASAGRANDE, H. Nova técnica para a determinação da lactose e sacarose no doce de leite. Revista do Instituto de Laticínios Cândido Tostes, v. 37, n. 222, p. 3-7, 1982.

YANG, D.; YING, Y. Applications of RAMAN spectroscopy in agricultural products and food analysis: A review. Applied Spectroscopy Reviews, v. 46, n. 7, p. 539-560, 2011. 